Article

\title{
Tuning the Photophysical Features of Self-Assembling Photoactive Polypeptides for Light-Harvesting
}

\author{
Maciej Michalik ${ }^{1,2}$, Mateusz Zbyradowski ${ }^{1}$, Heriyanto ${ }^{1,3}$ and Leszek Fiedor ${ }^{1, *(\mathbb{C}}$ \\ 1 Faculty of Biochemistry, Biophysics and Biotechnology, Jagiellonian University, Gronostajowa 7, \\ 30-387 Kraków, Poland; maciej.michalik@uj.edu.pl (M.M.); mateusz.zbyradowski@doctoral.uj.edu.pl (M.Z.); \\ oho.pigment@yahoo.com (H.) \\ 2 Institute of Physics, Jagiellonian University, Łojasiewicza 11, 30-428 Kraków, Poland \\ 3 Ma Chung Research Center for Photosynthetic Pigments, Ma Chung University, Villa Puncak Tidar N-01, \\ Malang 65151, Indonesia \\ * Correspondence: leszek.fiedor@uj.edu.pl; Tel.: +48-12-664-6358
}

Received: 7 October 2019; Accepted: 27 October 2019; Published: 30 October 2019

\begin{abstract}
The LH1 complex is the major light-harvesting antenna of purple photosynthetic bacteria. Its role is to capture photons, and then store them and transfer the excitation energy to the photosynthetic reaction center. The structure of LH1 is modular and it cooperatively self-assembles from the subunits composed of short transmembrane polypeptides that reversibly bind the photoactive cofactors: bacteriochlorophyll and carotenoid. LH1 assembly, the intra-complex interactions and the light-harvesting features of LH1 can be controlled in micellar media by varying the surfactant concentration and by adding carotenoid and/or a co-solvent. By exploiting this approach, we can manipulate the size of the assembly, the intensity of light absorption, and the energy and lifetime of its first excited singlet state. For instance, via the introduction of Ni-substituted bacteriochlorophyll into LH1, the lifetime of this electronic state of the antenna can be shortened by almost three orders of magnitude. On the other hand, via the exchange of carotenoid, light absorption in the visible range can be tuned. These results show how in a relatively simple self-assembling pigment-polypeptide system a sophisticated functional tuning can be achieved and thus they provide guidelines for the construction of bio-inspired photoactive nanodevices.
\end{abstract}

Keywords: LH1 antenna; solar energy conversion; spectral tuning; bacteriochlorophyll; carotenoid; reconstitution; pigment exchange; excitation energy transfer; oligomer size; pigment-pigment interactions

\section{Introduction}

The evolution of photosynthesis on Earth began approximately 3.5 billion years ago with the appearance of anaerobic phototrophic bacteria in the oceans, soon (in geological terms) followed by the emergence of oxygenic cyanobacteria, and then by plants [1]. Millions of years of efficient solar energy conversion by photosynthetic autotrophs resulted in a surplus of biomass and molecular oxygen. The accumulation of this very reactive by-product of oxygenic photosynthesis led to the oxidation of the planetary crust and the reductive primary atmosphere being turned into an oxidative one, saturated to a high degree with oxygen. As a result, evolution accelerated and all life forms could flourish throughout the planet [1]. Purple photosynthetic bacteria seem to be a direct descendent of these archaic phototrophic bacteria. Their relatively simple photosynthetic apparatus, composed of a photosynthetic reaction center $(\mathrm{RC})$ surrounded by a modular ring-shaped light-harvesting antenna 1 (LH1), forming together the so-called core complex (RC-LH1), is exceptional in many respects. 
It seems to be the oldest type of biological photodevice [2] that absorbs photons over a wide spectral range and with a high level of efficiency converts their energy into chemical energy. Bacterial RC is a prototype for RCs present in practically all other types of photosynthetic organisms [2]. In RC, which is the core of this photodevice, solar energy is used, with nearly $100 \%$ quantum efficiency, to drive electric charge separation across the photosynthetic membrane, which is then converted into chemically stable intermediates [3]. To achieve such a high level of performance, the photosynthetic apparatus underwent long-lasting evolution and can be regarded as having passed the "longest testing procedure", during which its design and functioning principles could be perfected.

In most purple bacteria, the RC-LH1 core complex is accompanied by the accessory (or peripheral) LH2 or LH3 antenna complexes, which are structurally similar to LH1 and function to enhance the active cross-section for light-harvesting (LH) [4-6]. Although the LH pigment-protein complexes in oxygenic phototrophs are more diverse, the majority of photosynthetic antennae share some key features because of the close similarity of the molecules they bind, carotenoids (Crts) and (bacterio)chlorophylls [7,8], and the role they serve- to fuel the RCs with excitations. Their overall performance is an interplay of numerous factors but there seems to exist no strict structural pattern for the LH antennae, and indeed high functional efficiency in LH can be achieved in several ways [7,9]. The LH1 antenna, directly associated with the RC, is remarkable in terms of its simplistic and modular but highly functional as well as aesthetically pleasing design (see Figure 1) [10,11]. It comprises 15-16 heterodimeric units composed of short hydrophobic $\alpha$ and $\beta$ polypeptides that bind two types of pigments: bacteriochlorophyll (BChl) and Crt $[10,12,13]$. The structures of BChl $a$ and spirilloxanthin (Spx), the native pigments present in LH1 from Rhodospirillum (Rsp.) rubrum, a model organism in photosynthetic research [12,14-16], are shown in Figure 1. BChla, which is the major functional (photoactive) and structural component of LH1, is a porphyrin-derived macrocyclic tetrapyrrole that binds $\mathrm{Mg}^{2+}$ in the central cavity. The pigment is highly hydrophobic and water-insoluble, owing to the presence of the side residue of phytyl [8]. The other component, $\mathrm{Crt}$, is a long-chain unsaturated isoprenoid that hosts an extensive system of delocalized $\pi$-electrons, formed by many conjugated double $\mathrm{C}-\mathrm{C}$ bonds. The primary role of $\mathrm{Crt}$ is the photoprotection of the antenna but it also functions in $\mathrm{LH}$ and contributes to structural stabilization of the entire pigment-protein ensemble of LH1 [17-19]. In the fully assembled LH1, each $\alpha-\beta$ heterodimer hosts a dimer of BChla molecules and a Crt molecule. The $\alpha-\beta$ heterodimers, assembled in a cylinder, serve as a scaffold for a large ensemble of BChl molecules [6,11], whose role is to efficiently capture photons, transiently store the excitation, and funnel the excitation energy to RC, where it is used to drive the photochemical reactions.

The formation of LH1, sometimes referred to as B880, from its monomeric (B780) and dimeric (B820) subunits in micellar medium ( $n$-octyl- $\beta$-glucopyranoside, $\beta$-OG) is schematically presented in Figure 1. The structure of LH1 is exceptional from both the mechanistic and engineering points of view because of its unique self-assembling property that allowed a pigment-protein complex to be completely reconstituted from the isolated components $[17,20]$. Like other photosynthetic pigment proteins, the polypeptides and $\mathrm{BChl}$ are indispensable for assembly, and importantly, information about the spatial organization of the system is encoded in their structures [21]. In effect, the LH1 system in a lipid environment is self-organizing, apparently with no need for external factors [18,20,22]. Our reconstitution approach takes advantage of this feature of LH1 $[18,22,23]$. In this paper, we show how it can be exploited to allow the essential functional and structural properties of a biological photodevice - the bacterial photosynthetic antenna LH1 — to be controlled and tuned. These design principles and traits of photosynthetic antenna LH1 may serve as inspiration for the engineering of biomimetic photodevices for solar energy conversion. 
A

$\boldsymbol{\alpha}$ MWRIWQLFDPRQALVGLATFLFVLALLIHFILLSTERFNWLEGASTKPVQTS

$\boldsymbol{\beta}$ EVKOESLSGITEGEAKEFHKIFTSSILVFFGVAAFAHLLVWIWRPWVPGPNGYS

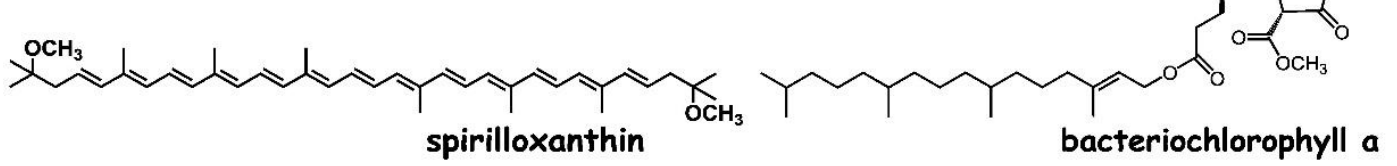

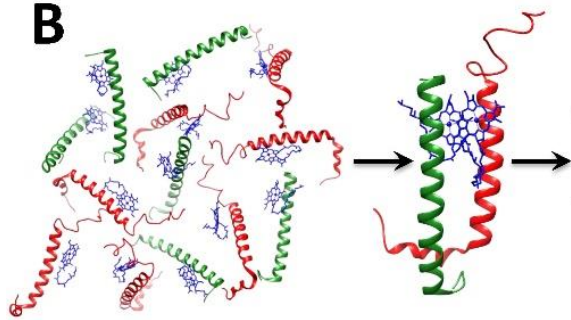

B820

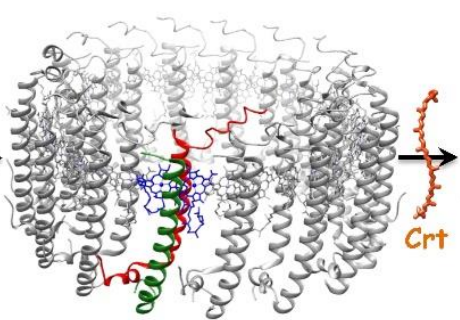

B870

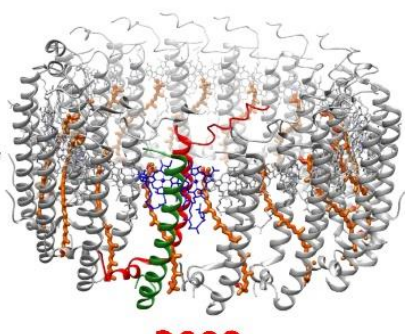

B880

Figure 1. (A) The amino-acid sequences and structural formulae of the components of the LH1 antenna from the purple photosynthetic bacterium Rhodospirillum rubrum. (B) A scheme showing the assembly of the carotenoidless (B870) and carotenoid-binding LH1 complex (B880) from the monomeric (B780) and dimeric (B820) subunits, and a carotenoid (Crt), in a micellar environment. The sizes of the complexes are not to scale. The structure of LH1 is taken from the Protein Data Bank (entry 5Y5S).

\section{Materials and Methods}

\subsection{Culturing of Bacteria and Isolation of Chromatophores}

The purple photosynthetic bacteria Rsp. rubrum S1 and Rhodobacter (Rdb.) sphaeroides 2.4.1 were cultivated anaerobically in the Cohen-Bazire medium [24] at $28^{\circ} \mathrm{C}$ under continuous illumination with fluorescent white light. The harvested cells were disrupted using a French press $(2 \times 15,000 \mathrm{psi})$ and the fraction of chromatophores was separated from the cell debris by centrifugation (50,000 $\mathrm{g}, 90 \mathrm{~min})$. The chromatophores were stored at $-30^{\circ} \mathrm{C}$ until use.

\subsection{Isolation of Pigments}

Spheroidene (Sph) was isolated from the cells of Rdb. sphaeroides 2.4.1 as described by Fujii et al. [25]. Water and BChla were extracted from the wet cells using cold methanol, and the Crts were then extracted using cold acetone. After fractionation against a saturated aqueous $\mathrm{NaCl}$ and $n$-hexane $(1: 1, v: v)$, the Crt extract was subjected to two rounds of column chromatography on alumina (ICN, activity II) equilibrated with $n$-hexane. Sph was eluted with 5-10\% (v:v) diethyl ether in $n$-hexane, vacuum dried and stored in a mixture of $n$-hexane and tetrahydrofuran $(4: 1, \mathrm{v}: \mathrm{v})$ at $-30^{\circ} \mathrm{C}$.

$\mathrm{BChl} a$ was isolated using the method described by Omata et al. [26]. A portion of freeze-dried cells of Rsp. rubrum S1 was extracted using cold methanol. The extract was dried under vacuum, dissolved in acetone, and loaded on a DEAE-Sepharose CL-6B (Sigma-Aldrich, St. Louis, MO, USA) column pre-equilibrated in acetone. The fraction of $\mathrm{BChl} a$ was eluted with $20 \%$ methanol in acetone (v:v), vacuum dried and stored at $-30{ }^{\circ} \mathrm{C}$ under Ar. All the solvents were of high performance liquid chromatography (HPLC) grade and purchased from Sigma (Sigma-Aldrich, St. Louis, MO, USA).

\subsection{Synthesis of Ni-bacteriopheophytin a}

Bacteriopheophytin a (BPheo) was prepared by demetalation of BChla in glacial acetic acid. The Ni-substituted BChla (Ni-BPheo) was obtained via transmetalation of a cadmium intermediate 
(Cd-BPheo) according to Hartwich et al. [27]. Cd-BPheo was synthesized from BPheo with anhydrous cadmium acetate in dimethylformamide. The precursor complex was then transferred to acetone and transmetalated in the presence of anhydrous $\mathrm{NiCl}_{2}$ to yield $\mathrm{Ni}-\mathrm{BPheo}$. The product was isolated using thin layer chromatography on Silica gel 60 (Merck, Darmstadt, Germany) using a 5\% acetone in toluene as an eluent. The purity of the pigments was confirmed spectrophotometrically and using analytical HPLC (see below).

\subsection{Isolation of Native LH1}

The native LH1 antenna from the Rsp. rubrum S1 strain was isolated as described previously [23]. Briefly, the bacterial chromatophores were solubilized in $0.45 \%(w / v)$ lauryldimethylamine $N$-oxide (LDAO, Sigma-Aldrich, St. Louis, MO, USA) in Tris-HCl buffer and separated from RC by ultracentrifugation. The pellet of the RC-depleted chromatophores was solubilized in $2.35 \%(w / v)$ $\beta$-OG (Carbosynth, Compton, UK) in $20 \mathrm{mM}$ Tris-HCl, pH 7.8, and loaded on a DEAE-cellulose (DE52, Whatman, Maidstone, UK) column pre-equilibrated in $0.8 \% \beta-\mathrm{OG}(w / v)$. The fraction of pure LH1 was eluted with $180-200 \mathrm{mM} \mathrm{NaCl}$ in $0.8 \%$-OG.

\subsection{Preparation of LH1 Subunits}

The LH1 subforms were prepared according to the methods described previously [22,28]. A portion of chromatophores from the Rsp. rubrum S1 strain was freeze-dried and depleted of Crts via repeated extraction with benzene, as described previously [23]. The Crt-depleted residue was titrated with aqueous $20 \% \beta-\mathrm{OG}(w / v)$ until B870 was completely dissociated to the B820 form. The insoluble impurities were removed via centrifugation $(6000 \mathrm{~g}, 45 \mathrm{~min})$ and a supernatant that contained crude B820 was loaded on a DEAE-Sepharose FF (Sigma-Aldrich, St. Louis, MO, USA) column equilibrated in a $20 \mathrm{mM}$ Tris- $\mathrm{HCl}$ buffer, $\mathrm{pH} 7.8$, containing $1 \%(w / v) \beta$-OG. The impurities and free BChla were removed with $10-50 \mathrm{mM} \mathrm{NaCl}$ in $1 \% \beta-\mathrm{OG}$ and the $\mathrm{B} 820$ fraction was eluted with $80 \mathrm{mM} \mathrm{NaCl}$ in $1 \% \beta$-OG. The monomeric B780 form was obtained by titrating B820 with $20 \% \beta$-OG to a final concentration of $\sim 5 \%(w / v)$, while the oligomeric B870 form was obtained from B820 by lowering the detergent concentration below its critical micelle concentration (CMC), to $0.4 \%(w / v)$.

\subsection{Pigment Exchange in LH1}

Sph was inserted into LH1 according to the method described by Fiedor et al. [23]. Briefly, a powder of Crt-depleted chromatophores was solubilized in $0.3 \%$ (v:v) LDAO in $20 \mathrm{mM}$ Tris-HCl buffer to obtain a mixture of B780, B820, and B870. The mixture was titrated with Sph dissolved in acetone until the maximum of the $Q_{Y}$ absorption band was shifted above $880 \mathrm{~nm}$. The reconstituted complex (Sph-LH1) was kept overnight at $4^{\circ} \mathrm{C}$ in the dark and then purified on a DEAE-cellulose column equilibrated in $0.03 \%$ (v:v) LDAO. Pure Sph-LH1 was eluted with $200 \mathrm{mM} \mathrm{NaCl}$ in $0.03 \%$ LDAO. To insert Ni-BPheo into LH1, a portion of Ni-BPheo dissolved in acetone was added to the B780-B820-B870 mixture before titration with Sph, following a previously published method [29].

To determine the pigment stoichiometry in LH1, the complex was loaded on a small DEAE-cellulose column equilibrated with $0.03 \%$ LDAO and thoroughly washed with distilled water to remove the detergent. The pigments were extracted with acetone, dried under vacuum, and the pigment composition of the extract was analyzed using HPLC on a $5 \mu \mathrm{m}$ LiChrospher Si-60 column (Merck) using a Varian ProStar (Varian, Palo Alto, CA, USA) system equipped with a TIDAS diode array detector (J\&M Analytik, Essingen, Germany) for on line measurements of the absorption spectra. The extract was loaded on the column in toluene and eluted with $98.9-95.9 \%$ toluene, $0.1 \%$ 2-propanol and $1-4 \%$ methanol (v:v) as described previously [13].

\subsection{Spectroscopic Measurements}

The electronic absorption and circular dichroism (CD) spectra were recorded in $1 \mathrm{~cm}$ quartz cuvettes using a Cary 60 Bio spectrophotometer (Varian, Palo Alto, CA, USA) and a J-815 spectropolarimeter 
(JASCO, Hachioji, Tokyo, Japan), respectively. The excitation and emission spectra were measured on a Fluoro Max-P fluorometer (Horiba Jobin Yvon, Kyoto, Japan), equipped with a cooled red-sensitive photomultiplier (R2658, Hamamatsu Photonics, Hamamatsu City, Japan) and a $690 \mathrm{~nm}$ cut-off filter. The fluorescence emission lifetime was measured using a time-domain Chronos $\mathrm{BH}$ fluorometer (ISS, Champaign, IL, USA), with a monochromator set to $900 \mathrm{~nm}$ and a H7422P-50 photomultiplier (Hamamatsu Photonics, Hamamatsu City, Japan) as a detector. The excitation pulses of 74 ps duration, output power of $157 \mathrm{~mW}$ and $20 \mathrm{MHz}$ repetition rate were generated by a $374 \mathrm{~nm}$ or $478 \mathrm{~nm}$ laser diode. The emission decay profiles were analyzed using the ISS Vinci software (version 2.0, ISS, Champaign, IL, USA). All measurements were taken at room temperature.

\section{Results}

\subsection{Isolation of Various Oligomeric Forms of LH1}

The subunits of LH1 of various sizes were obtained via detergent-induced dissociation of Crt-free LH1 (B870). The dimeric subunits B820 were isolated from the Crt-depleted chromatophores of wild-type Rsp. rubrum by titration with $\beta$-OG, until the $820 \mathrm{~nm}$ absorption band was saturated, at a final concentration of 3.2\% $\beta$-OG. The fraction of B820 subunits was isolated using ion-exchange chromatography on DEAE-Sepharose FF in $1 \% \beta$-OG [22]. By increasing the concentration of $\beta$-OG to $5 \%$, the B820 heterodimer was dissociated to the monomeric B780 subunits, comprised of single $\alpha$ and $\beta$ polypeptides, each coordinating a single $\mathrm{BChla}$ molecule [30]. The oligomeric $\mathrm{B} 870$ form was obtained via reassociation of $\mathrm{B} 820$ in $0.4 \% \beta$-OG. The association of a B780/B820 mixture in the presence of $\mathrm{Crts}$ (in LDAO) yields the B880 form (see below), which can be then purified chromatographically [23].

\subsection{Electronic Properties}

The electronic absorption spectra of the subunits and reassembled B870 complex are shown in Figure 2. The spectrum of $\mathrm{B} 780$ resembles that of monomeric $\mathrm{BChl} a$, while its dimerization to $\mathrm{B} 820$ markedly alters the absorption profile. All the absorption bands are red-shifted but to various degrees (Table 1), the Soret and $Q_{X}$ bands by several nanometers, while the $Q_{Y}$ transition moves from $780 \mathrm{~nm}$ to $820 \mathrm{~nm}$, and its intensity substantially increases. The association of B820 into B870 further lowers the energy of the $Q_{Y}$ transition $\left(870 \mathrm{~nm}\right.$ ) and its intensity remains high, whereas the Soret and $Q_{X}$ bands are less affected. If compared to $\mathrm{BChl} a$ in solution, the oscillator strength of the $\mathrm{BChl} a \mathrm{Q}_{\mathrm{Y}}$ transition increases upon oligomerization, by 50\% in B820 and 30\% in B870 (Figure 2). Oligomerization of the subunits is accompanied by changes in the $\mathrm{CD}$ spectra in the near-IR range (Figure 2). The $\mathrm{CD}$ features of B780 are weak but in the case of B820 and B870 very distinct features appear in their spectra, with strong but opposite non-conservative Cotton effects that have broad lobes centered at $792 \mathrm{~nm}$ and $830 \mathrm{~nm}$, and at $853 \mathrm{~nm}$ and $888 \mathrm{~nm}$, respectively.

Table 1. The absorption and emission maxima, and the first singlet excited-state lifetime $\left(\tau_{\mathrm{fl}}\right)$ of BChla in acetone, the monomeric (B780), dimeric (B820) and oligomeric (B870) subunits of LH1, the LH1 reconstituted with Sph (Sph-LH1) and the native LH1. The excitation wavelength was 374 or $478 \mathrm{~nm}$.

\begin{tabular}{cccccc}
\hline & \multicolumn{2}{c}{ Major Absorption Maxima $\left(\mathbf{n m}, \mathbf{c m}^{-\mathbf{1}}\right)$} & \multicolumn{2}{c}{$\begin{array}{c}\text { Fluorescence } \\
\text { Maximum }(\mathbf{n m})\end{array}$} & $\boldsymbol{\tau}_{\mathbf{f l}}(\mathbf{n s})$ \\
\cline { 2 - 4 } & Soret & $\mathbf{Q x}$ & $\mathbf{Q} \mathbf{Y}$ & 775 & $2.9^{a}$ \\
BChl $a$ & 357,28011 & 579,17271 & 770,12987 & 791 & $2.30 \pm 0.003$ \\
B780 & 361,27701 & 586,17065 & 780,12821 & 833 & $0.77 \pm 0.02$ \\
B820 & 370,27027 & 595,16807 & 821,12180 & 887 & $0.61 \pm 0.003$ \\
B870 & 375,26667 & 590,16950 & 869,11507 & 904 & $0.79 \pm 0.001$ \\
Sph-LH1 & 377,26525 & 591,16920 & 883,11325 & 902 & $0.85 \pm 0.02$ \\
native LH1 & 377,26525 & 590,16950 & 881,11351 &
\end{tabular}



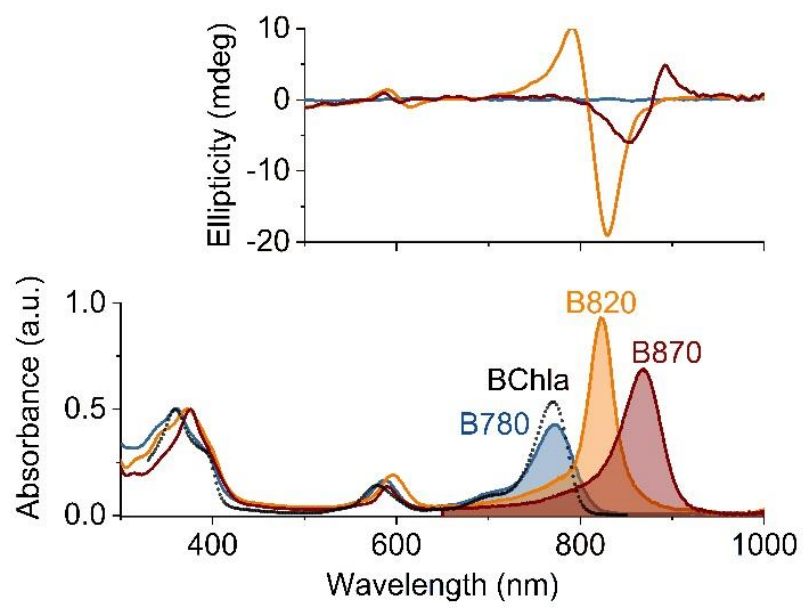

Figure 2. The electronic absorption (lower panel) and circular dichroism (upper panel) spectra of the B780 (blue), B820 (orange) and B870 (dark pink) oligomeric forms of LH1 in 5\%, $1 \%$ and $0.4 \%$ aqueous $\beta-\mathrm{OG}$, respectively, recorded at room temperature. The absorption spectra were normalized to the maximum of the Soret band whereas the circular dichroism spectra to the absorption intensity of the QY maximum. For comparison, also the absorption spectrum of monomeric bacteriochlorophyll a (BChla) in acetone is shown (black dotted line). The shaded areas denote the integrated intensities of the $Q_{Y}$ transitions.

\subsection{LH1 Assembly}

The progress of LH1 formation in the presence of Sph as monitored by electronic absorption spectroscopy is shown in Figure 3. The B780 and B820 subunits associate upon the addition of Sph and form B880, i.e., the Crt-binding LH1. The complex formation is complete when the QY transition shifts to $\sim 882 \mathrm{~nm}$ and the B780 and B820 absorption bands disappear. The absorption maxima of Sph (400-530 nm), from the first stages of reconstitution are red-shifted by $\sim 15 \mathrm{~nm}$ with respect to the maxima in acetone, and their positions stay constant during the folding of the antenna (Figure 3). The maxima of Sph absorption are located in the same positions as in the spectra of fully assembled and isolated LH1 [29,32]. The assembly of the subunits can also be induced in the absence of Crt, either by lowering the detergent concentration below its CMC value or by the use of a co-solvent, for instance acetone, and then the B870 complex is formed (not shown) [18].

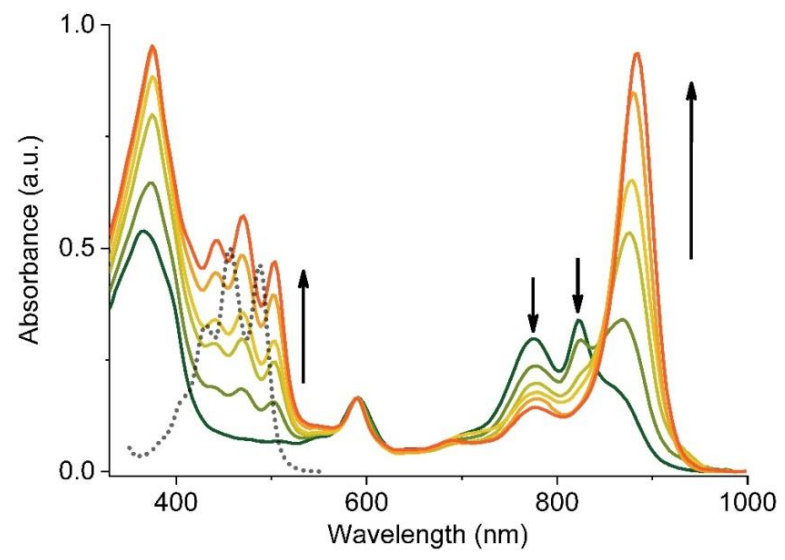

Figure 3. Spectral changes recorded during a Sph-induced reassembly of LH1 from its B780 and B820 subunits in micellar environment of LDAO. The spectra were normalized to the $Q_{X}$ band. The arrows indicate the direction of the changes in the spectra. For comparison, the absorption spectrum of Sph in acetone is also shown (black dotted line). 
The Crt-free LH1 antenna from Rsp. rubrum was used as a platform to introduce different Crts, also non-native to this species, into the identical BChla-polypeptide context. The absorption spectra in the visible range and the colors of the purified LH1 complexes which were reconstituted with five various Crts, i.e., neurosporene, spheroidene, lycopene, anhydrorhodovibrin and spirilloxanthin (native), are shown in Figure 4. With the increase in the number of double $\mathrm{C}-\mathrm{C}$ bonds contributing to the conjugated $\pi$-electron system, the Crt absorption bands shift to the red and the color of the antenna shifts from green to red (see the photograph in Figure 4). In effect, the Crt absorption bands fill the gap between the $\mathrm{BChl} a$ absorption bands.

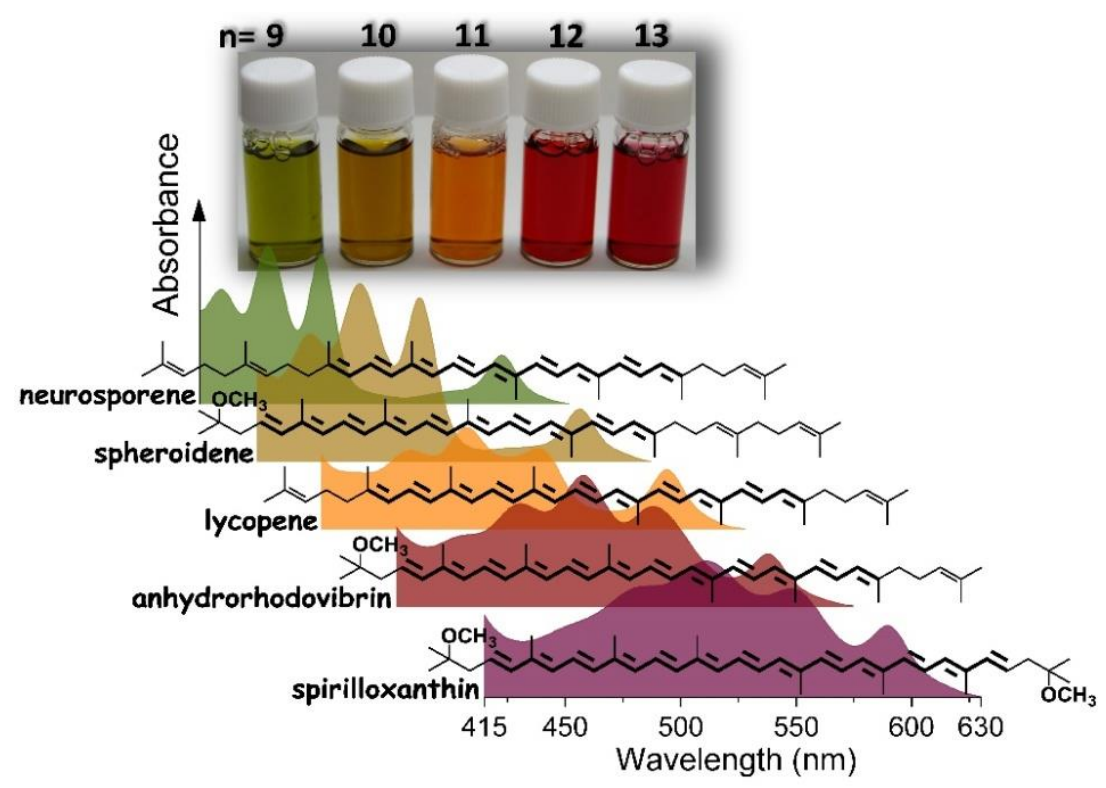

Figure 4. The electronic absorption spectra and coloration (the photograph in the upper part) of LH1 reconstituted with five different carotenoids with the length of their system of conjugated double $\mathrm{C}-\mathrm{C}$ bonds ( $n$ ) varying from 9 to 13 . The structural formulae of the carotenoids are shown above the corresponding absorption spectra.

\subsection{Intra-complex Energy Transfer}

The effects of the conjugation length in Crt on the efficiency of intra-complex $\mathrm{Crt} \rightarrow \mathrm{BChl}$ energy transfer were examined, comparing the native LH1, which contains Spx, and LH1 reconstituted with Sph (Sph-LH1). The LH capability of these two Crts, incorporated into the same BChla-polypeptide matrix, was determined using a previously described method [33], by comparing the intensities of the fractional absorption and fluorescence excitation spectra normalized to the $\mathrm{BChl} \mathrm{Q}_{\mathrm{X}}$ band (Figure 5). The ratio of spectral integrals between 430 and $560 \mathrm{~nm}$ gives an estimate of the efficiency of excitation energy transfer. In the native LH1, the efficiency of intra-complex excitation energy transfer was found to be $40 \% \pm 5 \%$, which agrees with the values previously reported $[16,17,34]$. In Sph-LH1 it is markedly higher, between $85 \%$ and $95 \%$ which is significantly different from $58-75 \%$ previously found in the LH1 from Rdb. sphaeroides which contained the same Crt, either native or reconstituted [17,34,35]. 


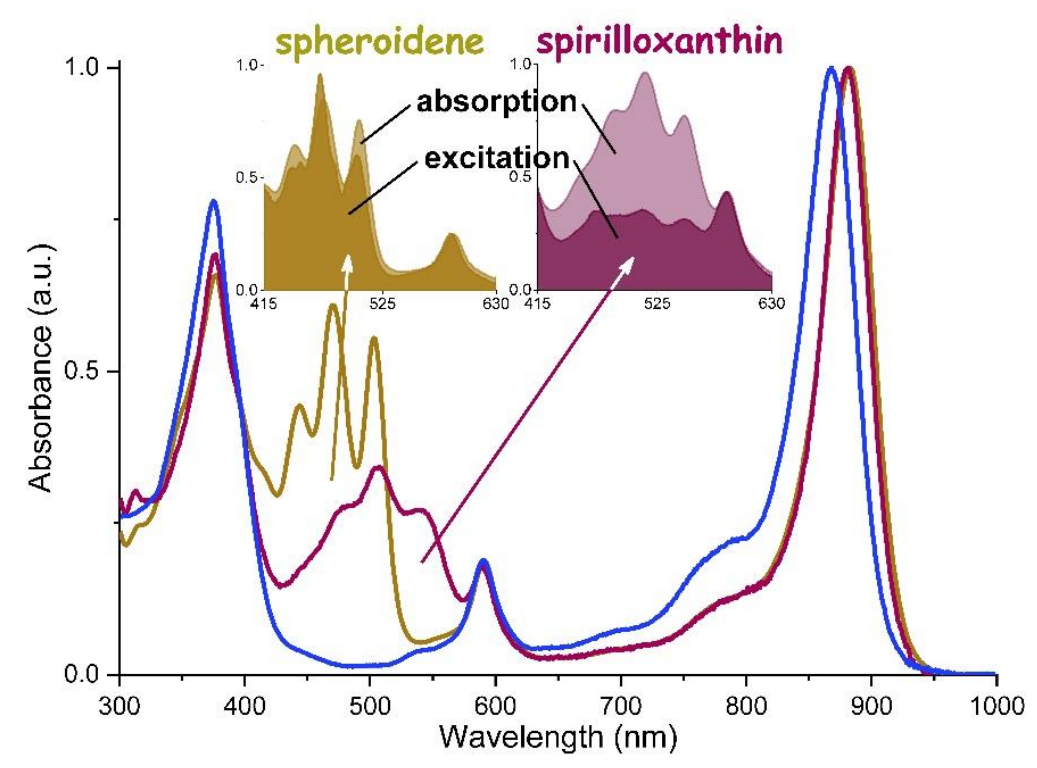

Figure 5. The electronic absorption spectra of B870 (blue line) and LH1 reconstituted either with spheroidene (dark yellow line) or spirilloxanthin (purple line). In the insert, the overlapped absorption and fluorescence excitation spectra of the two complexes are shown. The spectra were normalized to the $Q_{Y}$ band.

\subsection{Excited-State Properties}

To assess how the properties of the first excited singlet state of polypeptide-bound BChla change depending on the oligomerization state of the pigment, the fluorescence lifetime $\left(\tau_{\mathrm{fl}}\right)$ of LH1 and its subunits was determined using picosecond time-resolved fluorometry. The values of $\tau_{\mathrm{fl}}$ are similar to the one reported previously $[30,36]$ and very strongly depend on the degree of LH1 subunit oligomerization. The emission of fluorescence from the monomeric pigment is relatively long-lived (Table 1), with $\tau_{\mathrm{fl}}$ ranging from 2.3 to $2.9 \mathrm{~ns}$. In the oligomeric forms $\tau_{\mathrm{fl}}$ drastically decreases to a sub-ns range, between 600 and 800 ps.

The LH1 reconstitution technique facilitates the insertion of modified BChls into the antenna complex. This approach was used to partially replace the native pigment with its Ni-substituted analog, Ni-BPheo. This substitution does not lead to significant changes in the electronic absorption and CD spectra of LH1 (not shown), whereas its $\mathrm{S}_{1}$ state features change drastically, as illustrated in Figure 6. The emission is quenched completely when the content of Ni-BPheo in LH1 reaches $15 \%$ regarding the native $\mathrm{BChl} a$ (Figure $6 \mathrm{~A}$ ). At the same time, the emission decay profile becomes bi-exponential, which contrasts a mono-exponential decay in the non-substituted LH1 (Figure 6B). The single emission component detected in the latter case corresponds to a $\tau_{\mathrm{fl}}$ value of $790 \mathrm{ps}$. The same component $\left(\tau_{\mathrm{fl}} \sim 800 \mathrm{ps}\right)$ is found in the emission from LH1 containing Ni-BPheo, in addition to a very short-lived emission, with $\tau_{\mathrm{fl}}<1$ ps (below the time resolution of the apparatus). The contribution of the long-lived component decreases with the growing content of Ni-BPheo incorporated in LH1 (not shown). 
A

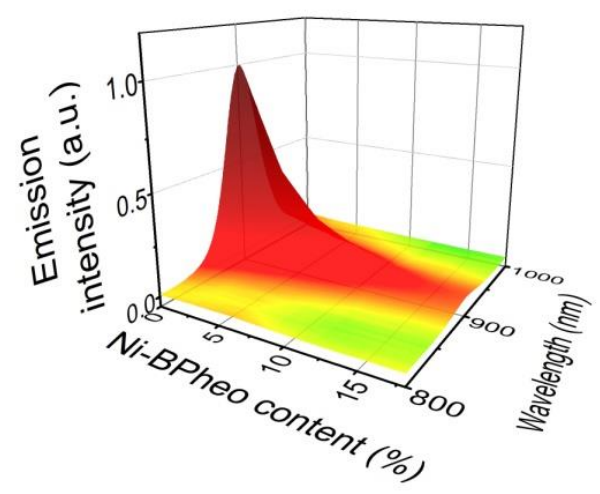

B

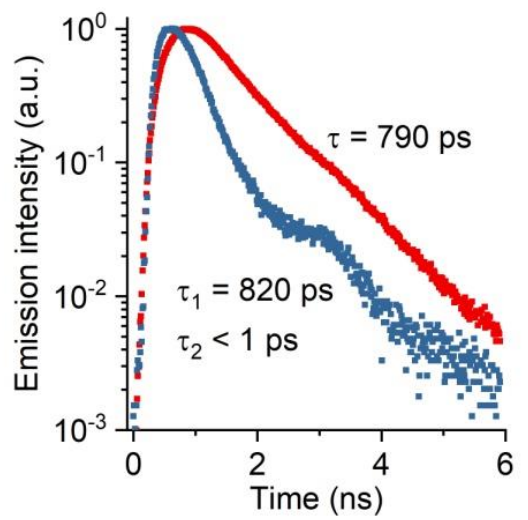

Figure 6. (A) The steady-state emission spectra of LH1 substituted with varying amounts of Ni-BPheo. (B) Fluorescence decay profiles of LH1 substituted with $0 \%$ (red trace) and 18\% (blue trace) of Ni-BPheo, with the fitted emission lifetime components. The traces were normalized to the maximum intensity. The optical density of the samples at the excitation wavelength $(478 \mathrm{~nm})$ was $0.1 \mathrm{~cm}^{-1}$.

\section{Discussion}

\subsection{Tuning of the Oligomeric State}

The equilibrium between the LH1 subunits B780 $\leftrightarrow$ B820 $\leftrightarrow$ B870/880 can be pushed towards oligomerization by enhancing the polypeptide-cofactor and polypeptide-polypeptide interactions, which can be achieved in several ways. At high concentrations of surfactant $(5 \% \beta-O G)$ the antenna is completely dissociated into the monomeric B780 subunits. Upon lowering of the surfactant concentration, the subunits dimerize to B820, which further associate to B870. The subunit oligomeric state is reflected in the characteristic position of the QY absorption band and shape of the CD spectrum, which facilitates a convenient monitoring of the oligomerization stages (Figure 3). The assembly of LH1 is accompanied by a gradual red-shift of the QY transition of BChl $a$ from $780 \mathrm{~nm}$ to $880 \mathrm{~nm}$, while in the CD spectrum a strong Cotton effect appears in the corresponding spectral regions, with the sign inverted when going from B820 to B870. Depending on the oligomer size, the oscillator strength of the QY transition of BChla increases by 50\% (B820) and 30\% (B870), which is indicative of an increase in the dipole strength of the lowest energy transition (see below).

The introduction of Crt and organic co-solvent into the reconstitution mixture also markedly affects the equilibrium between B780, B820, and B870/880. Crt triggers the assembly of B880 even at a very low Crt-to-subunit ratio, which indicates a high affinity of Crt to its binding sites and cooperativity of Crt-subunit association and cooperative folding of LH1 [23]. The red-shift of the absorption bands of LH1-bound Crt, as observed from the onset of reconstitution, also speaks for strong interactions between Crt and the $\alpha$ and $\beta$ polypeptides. The driving force for complex assembly is mostly provided by $\pi-\pi$ stacking between Crt and aromatic residues at the core of the helical transmembrane regions of the polypeptides [37], as well as via hydrophobic interactions [38]. Acetone, the co-solvent used during LH1 reconstitution, affects the properties of both the medium, by lowering its permittivity, and surfactant, by increasing the CMC value. In effect, electrostatic interactions between the interfaces of the transmembrane helical fragments of the $\alpha / \beta$ polypeptides are greatly intensified and the thermodynamics of LH1 assembly in a micellar medium is under the strong control of co-solvent and the Crt cofactor [18,38]. A similar stabilizing effect of Crts was found in LH2 [33]. In the absence of these external factors, the interaction energy $\left(\Delta \mathrm{H}^{\circ}\right)$ between the LH1 polypeptides equals $-580 \mathrm{~kJ} / \mathrm{mol}$. In the presence of acetone, $\Delta \mathrm{H}^{\circ}$ increases to $-1160 \mathrm{~kJ} / \mathrm{mol}$, and then to as much as $-1900 \mathrm{~kJ} / \mathrm{mol}$ in the presence of Crt. Intriguingly, despite these stabilization effects of Crt and co-solvent, and the increase in the inter-subunit interaction energy, the driving force for the association remains almost constant, apparently due to efficient enthalpy-entropy compensation in the system. 


\subsection{Tuning the Light-Harvesting and Excited-State Features}

The spectral range and the intensity of light absorption are among the most important aspects of LH using photosynthetic antenna complexes and in LH1 they can be controlled by varying the aggregation state of the subunits. Thus, the key photophysical features of $\mathrm{BChl} a$, its major chromophore, are tuned in such a way that the pigment $S_{1}$ state energy is lowered, which shifts the light absorption from the far-red edge of the visible range to the near-IR region. At the same time, due to hyperchromism, the cross-section for light absorption in this spectral region increases considerably. Two mechanisms seem to be responsible for such a sophisticated level of tuning available to the purple photosynthetic bacteria. The $\mathrm{S}_{1}$ energy is lowered due to excitonic interactions between BChla molecules, which in turn enables a certain intensity borrowing to the $\mathrm{S}_{0} \rightarrow \mathrm{S}_{1}$ transition from the higher energy transitions (see below) [36,39].

Light absorption in the blue-green spectral region can be tuned as well, via modifications of the Crt cofactor. As illustrated in Figure 4, this is achieved by varying $n$-the size of the delocalized $\pi$-electron system of Crt. In effect, the gap between the Soret and Qx transitions in BChla (see the absorption spectra in Figures 4 and 5), can be filled up with the absorption bands of Crts and this improves the capturing of photons in the visible range. However, this improvement comes at a certain cost, specifically the efficiency of the intra-complex $\mathrm{Crt} \rightarrow \mathrm{BChl}$ energy transfer is somewhat compromised. From almost $90 \%$, when the antenna binds Sph $(n=9)$, it drops to $\sim 30 \%$ in either native or reconstituted LH1 that contains Spx $(n=13)$. The most plausible reason for this dependence is the shortening of the lifetime of the $S_{2}$ donor state of $\mathrm{Crt}$ as $n$ is increasing [16,40]. Efficient and fast intra-complex Crt $\rightarrow$ $\mathrm{BChl}$ energy transfer takes place only if Crt is incorporated into properly folded LH1 [17] and hence its efficiency, being strongly dependent on the mutual orientation and inter-pigment distances [33], can be regarded as an indicator that the assembled antenna complex is functioning properly. A very high efficiency of energy transfer in Sph-LH1, reaching 90\%, confirms that a fully functional LH1 complex incorporating the non-native Crts has been reconstituted [16,23]. In this context, it should be noted that in the native photosynthetic antennae neither LH nor structural stabilization but the photoprotection seems to be the most important role of Crts [19].

The red-shift of the $Q_{Y}$ band and the characteristic shape of the CD spectra of B820 and B870 show exciton splitting of the energy levels of BChla in LH1, which is due to excitonic coupling in the ensembles of chromophores [41]. Such an interaction is observed when transition dipole moments of participating pigments are physically very close $(<10 \AA)$, and their mutual orientation is in head-to-tail geometry [42]. This implies a specific arrangement of BChls in B820, which is achieved through association between the $\alpha / \beta$ polypeptides, induced via ion-pairing and hydrogen-bonding of complementary $N$-terminal domains in each polypeptide [43]. $\pi-\pi$ stacking between paired BChls and pigment-polypeptide and polypeptide-polypeptide hydrophobic interactions, which involve the phytyl moiety of BChl and $\alpha$-helical core of the polypeptide, also promote dimerization/oligomerization $[18,43,44]$. The assembly of B820 subunits into a B870 complex brings about a further red-shift of the $Q_{Y}$ band and characteristic $\mathrm{CD}$ features in the near-IR region (Figure 2), which suggests that excitonic coupling is enhanced, involving both intra- and inter-dimer interactions that engage a larger number of chromophores. Such spectral changes reflect the formation of a supercomplex, i.e., a large array of electronically coupled $\mathrm{BChl} a$ molecules. The electronic excitations in such systems are delocalized over several pigment molecules [45]. Another indication of exciton delocalization is an increased radiative rate in a molecular ensemble [36], the so-called superradiance, which is manifested in the shortening of the $S_{1}$ state lifetime, as reflected in the fluorescence lifetimes of the oligomeric forms of BChla (see Table 1).

The interactions between pigment molecules and the $\alpha$ and $\beta$ polypeptides in the assembled antenna are responsible for the tuning of excitonic interactions, which is achieved by maintaining a specific spatial arrangement of chromophores. Due to such tuning of BChl by the polypeptides in LH1, the quenching effects that would be expected to occur at a high local concentration of the pigment are also diminished $[6,46]$. The same design principle is exploited in the peripheral LH2 complexes of 
purple bacteria for collecting solar energy following the excitation sink principle, whereas the excitonic coupling of chromophores in LH1 allow excitations to concentrate near the RCs [6].

The chemical engineering of (bacterio)chlorophylls opens further possibilities for the tuning and modifications of the photosynthetic antennae. A very useful seems to be the substitution of central $\mathrm{Mg}^{2+}$ ion with divalent transient metal ions, such as $\mathrm{Cu}^{2+}, \mathrm{Ni}^{2+}, \mathrm{Co}^{2+}, \mathrm{Pd}^{2+} \mathrm{or} \mathrm{Pt}^{2+}$, owing to their specific mode of bonding to the core nitrogen atoms. Due to the unfilled $\mathrm{d}$ shell, such cations form strong mixed coordination-covalent bonds in the central cavity of (bacterio)chlorophylls [47,48]. This brings about novel photophysical features in the metallosubstituted pigments [47,49], while their relevant chemical properties, such as axial ligand coordination, remain unchanged. This facilitates their interactions with pigment-binding polypeptides and enables pigment exchange in LH1. Owing to the exceptional mode of the central metal binding, the internal conversion and/or intersystem crossing in the metallocomplexes are strongly affected [50-52]. In the extreme case, radiationless decay of the excited singlet state of Ni-BPheo takes place in less than $100 \mathrm{fs}$, during which the electronic excitation is converted into heat with $100 \%$ efficiency [47], which may be particularly advantageous. Ni-BPheo readily replaces BChla in LH1 and, with an extremely short-lived $S_{1}$ state, it can be used as an ultrafast excitation trap to probe energy migration pathways and exciton delocalization in both LH1 [13,53] and RC complexes [54]. The transient femtosecond absorption measurements in the LH1 complexes that contain Ni-BPheo showed that exciton delocalization over BChl ensemble in LH1 is large and involves at least $10 \mathrm{BChl} a$ molecules. Furthermore, a single Ni-BPheo molecule per $20 \mathrm{BChl} a$ molecules suffices to entirely quench LH1 fluorescence [13]. A very similar quenching effect is seen in LH1 from Rsp. rubrum, reconstituted with varying amounts of Ni-BPheo (see Figure 6). The incorporation of the Ni-analog of BChla as the excitation trap drastically shortens the fluorescence emission lifetime of LH1 which gives rise to another level of control of the antenna excited states.

\section{Conclusions}

The self-organizing polypeptide-cofactor system of the LH1 antenna from purple photosynthetic bacteria is a prominent example of a tunable biological photodevice. The tuning in LH1 is multi-level and concerns almost every aspect of the system; from its size and subunit oligomerization state, through the thermodynamics of its assembly, to its photophysics and LH features. There are several ways to control the reversible assembly of LH1 in lipid/micellar media, by adding a Crt cofactor and/or a co-solvent, or varying the temperature or the concentration of surfactant. The changes in the LH1 subunit oligomerization state are paralleled by sophisticated tuning of the photophysical features of the major chromophore, $\mathrm{BChl} a$, to optimize the $\mathrm{LH}$ and excitation energy transfer by the antenna. The Crt exchange approach enables the LH capability of LH1 to be tuned to take better advantage of photons available in the visible spectral region and to improve the efficiency of the intra-complex excitation energy transfer, in addition to photoprotection of the system. Another level of LH1 tuning is feasible via chemical modifications and exchange of the cofactors.

The bacterial photosynthetic antenna LH1 is an exceptionally versatile model system in which the principles of tuning the key functional features can be demonstrated as well as investigated in detail. The system is self-organizing in the lipid environment and the information required for its assembly is encoded in the structures of its components. Very significantly, LH1 design facilitates sophisticated functional tuning of its chromophores, resulting in highly efficient LH over a broad spectral range, the excitation storage and its concentration towards $\mathrm{RC}$, and the photoprotection, which is crucial in a system with a continuous energy flow through. Obviously, however, the biosynthetic costs of such a photoactive biological device are very high. Its assembly in the bacterial photosynthetic membranes requires a concerted cooperation between its components-three different types of biochemical entities, each being the product of a different biosynthetic pathway. Despite that, LH1 provides us with numerous and challenging design guidelines for the construction of man-made photodevices for efficient solar energy harvesting and conversion. 
Author Contributions: L.F. and M.M. conceived the work. H. and M.M. carried out the experimental part. L.F., M.M. and M.Z. wrote the manuscript.

Funding: This research was funded by National Science Center in Poland, grant number 2014/13/N/NZ1/00057 to M.M. L.F. acknowledges the support from an internal grant No. N19/DBS/000009.

Conflicts of Interest: The authors declare no conflict of interest.

\section{References}

1. Hohmann-Marriott, M.F.; Blankenship, R.E. Evolution of Photosynthesis. Annu. Rev. Plant Biol. 2011, 62, 515-548. [CrossRef]

2. Orf, G.S.; Gisriel, C.; Redding, K.E. Evolution of photosynthetic reaction centers: Insights from the structure of the heliobacterial reaction center. Photosynth. Res. 2018, 138, 11-37. [CrossRef]

3. Jones, M.R. Reaction centers: Structure and mechanism. In Light Harvesting in Photosynthesis; Croce, R., van Grondelle, R., van Amerongen, H., van Stokkum, I., Eds.; CRC Press: Boca Raton, FL, USA, 2018; pp. $181-205$.

4. Cogdell, R.J.; Gardiner, A.T.; Roszak, A.W.; Law, C.J.; Southall, J.; Isaacs, N.W. Rings, ellipses and horseshoes: How purple bacteria harvest solar energy. Photosynth. Res. 2004, 81, 207-214. [CrossRef] [PubMed]

5. Gabrielsen, M.; Gardiner, A.T.; Cogdell, R.J. Peripheral Complexes of Purple Bacteria. In The Purple Phototrophic Bacteria; Hunter, C.N., Daldal, F., Thurnauer, M.C., Beatty, J.T., Eds.; Springer Netherlands: Dordrecht, The Netherlands, 2009; pp. 135-153.

6. Mothersole, D.J.; Farmer, D.A.; Hitchcock, A.; Hunter, C.N. Photosynthetic apparatus in purple bacteria. In Light Harvesting in Photosynthesis; Croce, R., van Grondelle, R., van Amerongen, H., van Stokkum, I., Eds.; CRC Press: Boca Raton, FL, USA, 2018; pp. 95-120.

7. Croce, R.; van Amerongen, H. Natural strategies for photosynthetic light harvesting. Nat. Chem. Biol. 2014, 10, 492-501. [CrossRef] [PubMed]

8. Fiedor, L.; Zbyradowski, M.; Pilch, M. Tetrapyrrole pigments of photosynthetic antennae and reaction centers of higher plants: Structures, biophysicis, functions, biochemistry, mechanisms of regulation, applications. In Metabolism, Structure and Function of Plant Tetrapyrroles: Introduction, Microbial and Eukaryotic Chlorophyll Synthesis and Catabolism, 1st ed.; Grimm, B., Ed.; Academic Press: London, UK, 2019; Volume 90, pp. 1-33.

9. Mirkovic, T.; Ostroumov, E.E.; Anna, J.M.; Van Grondelle, R.; Scholes, G.D. Light absorption and energy transfer in the antenna complexes of photosynthetic organisms. Chem. Rev. 2017, 117, 249-293. [CrossRef] [PubMed]

10. Roszak, A.W.; Howard, T.D.; Southall, J.; Gardiner, A.T.; Law, C.J.; Isaacs, N.W.; Cogdell, R.J. Crystal structure of the RC-LH1 core complex from Rhodopseudomonas palustris. Science 2003, 302, 1969-1972. [CrossRef] [PubMed]

11. Niwa, S.; Yu, L.J.; Takeda, K.; Hirano, Y.; Kawakami, T.; Wang-Otomo, Z.Y.; Miki, K. Structure of the LH1-RC complex from Thermochromatium tepidum at 3.0 angstrom. Nature 2014, 508, 228-232. [CrossRef] [PubMed]

12. Karrasch, S.; Bullough, P.A.; Ghosh, R. The 8.5 A projection map of the light-harvesting complex from Rhodospirillum rubrum reveals a ring composed of 16 subunits. EMBO J. 1995, 14, 631-638. [CrossRef] [PubMed]

13. Fiedor, L.; Leupold, D.; Teuchner, K.; Voigt, B.; Hunter, C.N.; Scherz, A.; Scheer, H. Excitation trap approach to analyze size and pigment-pigment coupling: Reconstitution of LH1 antenna of Rhodobacter sphaeroides with Ni-substituted bacteriochlorophyll. Biochemistry 2001, 40, 3737-3747. [CrossRef]

14. Loach, P.A.; Sekura, D.L. Primary photochemistry and electron transport in Rhodospirillum rubrum. Biochemistry 1968, 7, 2642-2649. [CrossRef]

15. Picorel, R.; Belanger, G.; Gingras, G. Antenna holochrome B880 of Rhodospirillum rubrum S1. Pigment, phospholipid, and polypeptide composition. Biochemistry 1983, 22, 2491-2497. [CrossRef]

16. Akahane, J.; Rondonuwu, F.S.; Fiedor, L.; Watanabe, Y.; Koyama, Y. Dependence of singlet-energy transfer on the conjugation length of carotenoids reconstituted into the LH1 complex from Rhodospirillum rubrum G9. Chem. Phys. Lett. 2004, 393, 184-191. [CrossRef]

17. Davis, C.M.; Bustamante, P.L.; Loach, P.A. Reconstitution of the bacterial core light-harvesting complexes of Rhodobacter sphaeroides and Rhodospirillum rubrum with isolated $\alpha$ - and $\beta$-polypeptides, bacteriochlorophyll a, and carotenoid. J. Biol. Chem. 1995, 270, 5793-5804. [CrossRef] 
18. Fiedor, J.; Pilch, M.; Fiedor, L. Tuning the thermodynamics of association of transmembrane helices. J. Phys. Chem. B 2009, 113, 12831-12838. [CrossRef] [PubMed]

19. Polivka, T.; Frank, H. Light harvesting by carotenoids. Acc. Chem. Res. 2010, 43, 1125-1134. [CrossRef] [PubMed]

20. Parkes-Loach, P.S.; Sprinkle, J.R.; Loach, P.A. Reconstitution of the B873 light-harvesting complex of Rhodospirillum rubrum from separately isolated $\alpha$ - and $\beta$-polypeptides and bacteriochlorophyll a. Biochemistry 1988, 27, 2718-2727. [CrossRef] [PubMed]

21. Popot, J.-L.; Gerchman, S.-E.; Engelman, D.M. Refolding of bacteriorhodopsin in lipid bilayers. A thermodynamically controlled two-stage process. J. Mol. Biol. 1987, 198, 655-676. [CrossRef]

22. Fiedor, L.; Scheer, H. Trapping of an intermediate of LH1 complex assembly beyond the B820 subunit. J. Biol. Chem. 2005, 280, 20921-20926. [CrossRef]

23. Fiedor, L.; Akahane, J.; Koyama, Y. Carotenoid-induced cooperative formation of bacterial photosynthetic LH1 complex. Biochemistry 2004, 43, 16487-16496. [CrossRef]

24. Cohen-Bazire, G.; Sistrom, W.R.; Stanier, R.Y. Kinetic studies of pigment synthesis by non-sulphur purple bacteria. J. Cell. Comp. Physiol. 1957, 49, 25-58. [CrossRef]

25. Fujii, R.; Onaka, K.; Kuki, M.; Koyama, Y.; Watanabe, Y. The 2Ag- energies of all-trans-neurosporene and spheroidene as determined by fluorescence spectroscopy. Chem. Phys. Lett. 1998, 288, 847-853. [CrossRef]

26. Omata, T.; Murata, N. Preparation of chlorophyll a, chlorophyll b and bacteriochlorophyll a by column chromatography with DEAE-Sepharose CL-6B and Sepharose CL-6B. Plant Cell Physiol. 1983, 24, 1093-1100.

27. Hartwich, G.; Fiedor, L.; Simonin, I.; Cmiel, E.; Schäfer, W.; Noy, D.; Scherz, A.; Scheer, H. Metal-Substituted Bacteriochlorophylls. 1. Preparation and Influence of Metal and Coordination on Spectra. J. Am. Chem. Soc. 1998, 120, 3675-3683. [CrossRef]

28. Fiedor, L. Hexacoordination of bacteriochlorophyll in photosynthetic antenna LH1. Biochemistry 2006, 45, 1910-1918. [CrossRef]

29. Lambrev, P.H.; Miloslavina, Y.; Van Stokkum, I.H.M.; Stahl, A.D.; Michalik, M.; Susz, A.; Tworzydło, J.; Fiedor, J.; Huhn, G.; Groot, M.-L.; et al. Excitation energy trapping and dissipation by Ni-substituted bacteriochlorophyll a in reconstituted LH1 complexes from Rhodospirillum rubrum. J. Phys. Chem. B 2013, 117, 11260-11271. [CrossRef]

30. Chang, M.C.; Callahan, P.M.; Parkes-Loach, P.S.; Cotton, T.M.; Loach, P.A. Spectroscopic characterization of the light-harvesting complex of Rhodospirillum rubrum and its structural subunit. Biochemistry 1990, 29, 421-429. [CrossRef]

31. Karcz, D.; Boron, B.; Matwijczuk, A.; Furso, J.; Staron, J.; Ratuszna, A.; Fiedor, L. Lessons from chlorophylls: Modifications of porphyrinoids towards optimized solar energy conversion. Molecules 2014, 19, 15938-15954. [CrossRef]

32. Nakagawa, K.; Suzuki, S.; Fujii, R.; Gardiner, A.T.; Cogdell, R.J.; Nango, M.; Hashimoto, H. Probing the effect of the binding site on the electrostatic behavior of a series of carotenoids reconstituted into the light-harvesting 1 complex from purple photosynthetic bacterium Rhodospirillum rubrum detected by Stark spectroscopy. J. Phys. Chem. B 2008, 112, 9467-9475. [CrossRef]

33. Ashikhmin, A.; Makhneva, Z.; Bolshakov, M.; Moskalenko, A. Incorporation of spheroidene and spheroidenone into light-harvesting complexes from purple sulfur bacteria. J. Photochem. Photobio. B 2017, 170, 99-107. [CrossRef]

34. Cogdell, R.J.; Andersson, P.O.; Gillbro, T. Carotenoid singlet-states and their involvement in photosynthetic light-harvesting. J. Photochem. Photobiol. B 1992, 15, 105-112. [CrossRef]

35. Kramer, H.J.M.; Pennoyer, J.D.; van Grondelle, R.; Westerhuis, W.H.J.; Niederman, R.A.; Amesz, J. Low-temperature optical properties and pigment organization of the B875 light-harvesting bacteriochlorophyll-protein complex of purple photosynthetic bacteria. Biochim. Biophys. Acta 1984, 767, 335-344. [CrossRef]

36. Monshouwer, R.; Abrahamsson, M.; van Mourik, F.; van Grondelle, R. Superradiance and exciton delocalization in bacterial photosynthetic light-harvesting systems. J. Phys. Chem. B 1997, 101, 7241-7248. [CrossRef]

37. Wang, Z.-Y.; Muraoka, Y.; Shimonaga, M.; Kobayashi, M.; Nozawa, T. Selective detection and assignment of the solution NMR signals of bacteriochlorophyll a in a reconstituted subunit of a light-harvesting complex. J. Am. Chem. Soc. 2002, 124, 1072-1078. [CrossRef] [PubMed] 
38. Sturgis, J.N.; Robert, B. Thermodynamics of membrane polypeptide oligomerization in light-harvesting complexes and associated structural changes. J. Mol. Biol. 1994, 238, 445-454. [CrossRef] [PubMed]

39. Visschers, R.W.; Chang, M.C.; van Mourik, F.; Parkes-Loach, P.S.; Heller, B.A.; Loach, P.A.; van Grondelle, R. Fluorescence polarization and low-temperature absorption spectroscopy of a subunit form of light-harvesting complex I from purple photosynthetic bacteria. Biochemistry 1991, 30, 5734-5742. [CrossRef] [PubMed]

40. Noguchi, T.; Hayashi, H.; Tasumi, M. Factors controlling the efficiency of energy transfer from carotenoids to bacteriochlorophyll in purple photosynthetic bacteria. Biochim. Biophys. Acta 1990, 1017, 280-290. [CrossRef]

41. Van Amerongen, H.; Valkunas, L.; Van Grondelle, R. Photosynthetic Excitons; Farrer Road 912805; World Scientific: Singapore, 2000. [CrossRef]

42. Hashimoto, H.; Uragami, C.; Yukihira, N.; Gardiner, A.T.; Cogdell, R.J. Understanding/unravelling carotenoid excited singlet states. Interface 2018, 15, 20180026. [CrossRef]

43. Meadows, K.A.; Iida, K.; Tsuda, K.; Recchia, P.A.; Heller, B.A.; Antonio, B.; Nango, M.; Loach, P.A. Enzymatic and chemical cleavage of the core light-harvesting polypeptides of photosynthetic bacteria-Determination of the minimal polypeptide size and structure required for subunit and light-harvesting complex-formation. Biochemistry 1995, 34, 1559-1574. [CrossRef]

44. Parkes-Loach, P.S.; Majeed, A.P.; Law, C.J.; Loach, P.A. Interactions stabilizing the structure of the core light-harvesting complex (LH1) of photosynthetic bacteria and its subunit (B820). Biochemistry 2004, 43, 7003-7016. [CrossRef]

45. Dracheva, T.V.; Novoderezhkin, V.I.; Razjivin, A.P. Exciton delocalization in the light-harvesting LH2 complex of photosynthetic purple bacteria. Photochem. Photobiol. 1997, 66, 605-610. [CrossRef]

46. Drews, G. Formation of the light-harvesting complex I (B870) of anoxygenic phototrophic purple bacteria. Arch. Microbiol. 1996, 166, 151-159. [CrossRef] [PubMed]

47. Pilch, M.; Dudkowiak, A.; Jurzyk, B.; Łukasiewicz, J.; Susz, A.; Stochel, G.; Fiedor, L. Molecular symmetry determines the mechanism of a very efficient ultrafast excitation-to-heat conversion in Ni-substituted chlorophylls. Biochim. Biophys. Acta 2013, 1827, 30-37. [CrossRef] [PubMed]

48. Kania, A.; Pilch, M.; Rutkowska-Zbik, D.; Susz, A.; Stochel, G.; Fiedor, L. High-pressure and theoretical studies reveal significant differences in the electronic structure and bonding of magnesium, zinc and nickel ions in metalloporphyrinoids. Inorg. Chem. 2014, 53, 8473-8484. [CrossRef] [PubMed]

49. Kotkowiak, M.; Dudkowiak, A.; Fiedor, L. Intrinsic photoprotective mechanisms in chlorophylls. Angew. Chem. Int. Ed. 2017, 56, 10457-10461. [CrossRef]

50. Noy, D.; Fiedor, L.; Hartwich, G.; Scheer, H.; Scherz, A. Metal-substituted bacteriochlorophylls. 2. Changes in redox potentials and electronic transition energies are dominated by intramolecular electrostatic interactions. J. Am. Chem. Soc. 1998, 120, 3684-3693. [CrossRef]

51. Musewald, C.; Hartwich, G.; Lossau, H.; Gilch, P.; Pöllinger-Dammer, F.; Scheer, H.; Michel-Beyerle, M.E. Ultrafast photophysics and photochemistry of [Ni]-bacteriochlorophyll a. J. Phys. Chem. B 1999, 103, 7055-7060. [CrossRef]

52. Drzewiecka-Matuszek, A.; Skalna, A.; Karocki, A.; Stochel, G.; Fiedor, L. Effects of heavy central metal on the ground and excited states of chlorophyll. J. Biol. Inorg. Chem. 2005, 10, 453-462. [CrossRef]

53. Fiedor, L.; Scheer, H.; Hunter, C.N.; Tschirschwitz, F.; Voigt, B.; Ehlert, J.; Nibbering, E.; Leupold, D.; Elsaesser, T. Introduction of a $60 \mathrm{fs}$ deactivation channel in the photosynthetic antenna LH1 by Ni-Bacteriopheophytin a. Chem. Phys. Lett. 2000, 319, 145-152. [CrossRef]

54. Hartwich, G.; Scheer, H.; Aust, V.; Angerhofer, A. Absorption and ADMR studies on bacterial photosynthetic reaction centres with modified pigments. Biochim. Biophys. Acta 1995, 1230, 97-113. [CrossRef]

(C) 2019 by the authors. Licensee MDPI, Basel, Switzerland. This article is an open access article distributed under the terms and conditions of the Creative Commons Attribution (CC BY) license (http://creativecommons.org/licenses/by/4.0/). 